Research article Open Access

\title{
Haploinsufficiency for p190B RhoGAP inhibits MMTV-Neu tumor progression
}

\author{
Brandy M Heckman-Stoddard1,2, Tracy Vargo-Gogola ${ }^{3}$, Peter R McHenry ${ }^{3}$, Vivian Jiang ${ }^{1}$, \\ Matthew P Herrick ${ }^{1}$, Susan G Hilsenbeck ${ }^{4}$, Jeffrey Settleman ${ }^{5}$ and Jeffrey M Rosen ${ }^{1}$
}

\begin{abstract}
1Department of Molecular and Cellular Biology, Baylor College of Medicine, One Baylor Plaza, Houston, TX 77030, USA
${ }^{2}$ Cancer Prevention Fellowship Program, Center for Cancer Training, National Cancer Institute, 6120 Executive Boulevard, Bethesda, MD 20852, USA

3Department of Biochemistry and Molecular Biology, Indiana University School of Medicine, 1234 Notre Dame Avenue, South Bend, IN 4661 7, USA ${ }^{4}$ Lester and Sue Smith Breast Center and Department of Medicine, Baylor College of Medicine, One Baylor Plaza, Houston, TX 77030, USA

5Massachusetts General Hospital Cancer Center and Harvard Medical School, 13th Street, Charlestown, MA 02129, USA
\end{abstract}

Corresponding author: Jeffrey M Rosen, jrosen@bcm.edu

Received: 26 Feb 2009 Revisions requested: 14 Apr 2009 Revisions received: 21 Jul 2009 Accepted: 24 Aug 2009 Published: 24 Aug 2009

Breast Cancer Research 2009, 11:R61 (doi:10.1186/bcr2352)

This article is online at: http://breast-cancer-research.com/content/11/4/R61

(c) 2009 Heckman-Stoddard et al.; licensee BioMed Central Ltd.

This is an open access article distributed under the terms of the Creative Commons Attribution License (http://creativecommons.org/licenses/by/2.0), which permits unrestricted use, distribution, and reproduction in any medium, provided the original work is properly cited.

\begin{abstract}
Introduction Rho signaling regulates key cellular processes including proliferation, survival, and migration, and it has been implicated in the development of many types of cancer including breast cancer. P190B Rho GTPase activating protein (RhoGAP) functions as a major inhibitor of the Rho GTPases. $\mathrm{P} 190 \mathrm{~B}$ is required for mammary gland morphogenesis, and overexpression of $\mathrm{p} 190 \mathrm{~B}$ in the mammary gland induces hyperplastic lesions. Hence, we hypothesized that $\mathrm{p} 190 \mathrm{~B}$ may play a pivotal role in mammary tumorigenesis.
\end{abstract}

Methods To investigate the effects of loss of $p 190 \mathrm{~B}$ function on mammary tumor progression, $\mathrm{p} 190 \mathrm{~B}$ heterozygous mice were crossed with an MMTV-Neu breast cancer model. Effects of p190B deficiency on tumor latency, multiplicity, growth, preneoplastic progression and metastasis were evaluated. To investigate potential differences in tumor angiogenesis between the two groups, immunohistochemistry to detect von Willebrand factor was performed and quantified. To examine gene expression of potential mediators of the angiogenic switch, an angiogenesis PCR array was utilized and results were confirmed using immunohistochemistry. Finally, reciprocal transplantation of tumor fragments was performed to determine the impact of stromal deficiency of $\mathrm{p} 190 \mathrm{~B}$ on tumor angiogenesis.

Results $\mathrm{P} 190 \mathrm{~B}$ deficiency reduced tumor penetrance $(53 \%$ of


tumors) and markedly delayed tumor onset by an average of 46 weeks. Tumor multiplicity was also decreased, but an increase in the number of preneoplastic lesions was detected indicating that $\mathrm{p} 190 \mathrm{~B}$ deficiency inhibited preneoplastic progression. Angiogenesis was decreased in the p190B heterozygous tumors, and expression of a potent angiogenic inhibitor,

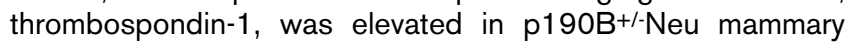


wild-type recipients restored tumor angiogenesis. Strikingly,



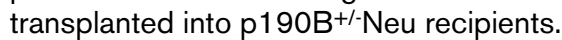

Conclusions These data suggest that p190B haploinsufficiency in the epithelium inhibits MMTV-Neu tumor initiation. Furthermore, p190B deficiency in the vasculature is responsible, in part, for the inhibition of MMTV-Neu tumor progression.

\section{Introduction}

Small GTPases of the Rho family act as nodes of signal transduction, integrating extracellular signals to affect actin cytoskeletal organization, cell adhesion, polarity, proliferation and migration, which are all important processes that become deregulated during cancer progression. Rho proteins are molecular switches that cycle between an active GTP-bound state and an inactive GDP-bound state, and their activities are spatially and temporally regulated. Three classes of proteins control Rho activity, including guanine nucleotide exchange

BSA: bovine serum albumin; GAP: GTPase activating protein; GTPases: guanine nucleotide triphosphatases; MMTV: mouse mammary tumor virus; Neu: rat homolog of ErbB2; PAK: p21-activated kinase; PCR: polymerase chain reaction; Rho: Ras homologous; ROK: Rho kinase; siRNA: small interfering RNA; TBS-1: thrombospondin-1; VWF: von Willebrand factor. 
factors that activate the switch, GTPase activating proteins (GAPs) that inactivate the switch, and guanine nucleotide dissociation inhibitors that prevent the exchange of GDP for GTP on the endogenous RhoGTPases [1-3]. Several studies have shown overexpression of Rho family members in human breast cancer samples by immunohistochemistry, by mutational analysis, or by RNA expression profiling [4-7]. The role of Rho signaling in breast cancer, however, is not well understood.

To elucidate the contribution of the Rho signaling pathway to the growth and progression of breast cancer, we focused on understanding the role of $\mathrm{p} 190 \mathrm{~B}$ RhoGAP, which has been shown previously to play an important role in normal mammary gland development $[8,9]$. P190B is a member of the RhoGAP family, which acts as negative regulator of Rho activity. The GAP domain of p190B demonstrated activity against Rho, Rac and Cdc42 [10], and more recently p190B was shown to directly bind Rnd3, Rac1 and RhoA [11]. Homozygous deletion of $\mathrm{p} 190 \mathrm{~B}$ results in central nervous system and lung defects, leading to perinatal lethality [12]. P190B-deficient embryos and cells are smaller than their wild-type counterparts, due in part to impaired insulin-like growth factor signaling. Importantly, the $\mathrm{p} 190 \mathrm{~B}$ and insulin-like growth factor signaling pathways have been shown to directly interact through Rho kinase (ROK) phosphorylation of insulin receptor substrate proteins and to be critically involved in regulating both cell growth and differentiation [12,13].

P190B is highly expressed throughout embryonic and virgin mammary gland development, with expression decreasing during late pregnancy and remaining low during lactation $[14,15]$. P190B homozygous-deficient mammary glands fail to undergo ductal morphogenesis. Moreover, loss of one allele of p190B transiently delays ductal morphogenesis and results in decreased proliferation within the terminal end buds, which drive ductal penetration into the fat pad [8]. Overexpression of p190B during virgin development disrupts terminal end bud morphogenesis, increases side branching, and delays ductal elongation, while overexpression during pregnancy results in hyperplastic lesions [9].

To elucidate the role of $\mathrm{p} 190 \mathrm{~B}$ in mammary tumor progression, we crossed p190B heterozygous mice with a mouse mammary tumor virus (MMTV)-Neu mouse model of breast cancer and examined multiple stages of tumor progression. Neu/ ErbB2 is a member of the epidermal growth factor receptor family that is amplified and overexpressed in 20 to $30 \%$ of human breast cancers and correlates with poor prognosis [16]. Several mouse models have been generated to examine mechanisms involved in ErbB2-mediated mammary tumor progression [17]. For these studies, we chose to use the MMTVNeu model that overexpresses a wild-type $\mathrm{Neu}$ proto-oncogene [18]. MMTV-Neu female mice develop preneoplastic lesions, some of which progress to adenocarcinomas, with a median onset of 7 months of age. Approximately 70 to $80 \%$ of tumor-bearing mice develop metastasic disease [18]. GAPs and related $\mathrm{G}$ proteins are reportedly upregulated in these tumors, suggesting that Rho signaling is important for MMTVNeu tumor formation and progression [19].

Using this approach, we demonstrate that $\mathrm{p} 190 \mathrm{~B}$ plays a critical role in MMTV-Neu-induced tumorigenesis. Strikingly, loss of one allele of $\mathrm{p} 190 \mathrm{~B}$ decreased tumor penetrance, delayed tumor onset, and reduced metastasis. Furthermore, the progression of preneoplastic lesions was inhibited by the loss of $\mathrm{p} 190 \mathrm{~B}$, and $\mathrm{p} 190 \mathrm{~B}$ heterozygous tumors have a reduced vascular network, thus implicating $\mathrm{p} 190 \mathrm{~B}$ in tumor angiogenesis. Reciprocal transplantation of tumor fragments confirmed that p190B expression in the stroma plays an important role in tumor angiogenesis. The effects of $\mathrm{p} 190 \mathrm{~B}$ loss on angiogenesis may be due in part to increased expression of a potent anti-angiogenic factor, thrombospondin-1 (TBS-1). The reduction in angiogenesis may contribute to the inhibition of metastasis.

\section{Materials and methods Mice strains and tumor analysis}

P190B null mice [12] were backcrossed to FVB for four generations. P190B+/- (FVB4) mice were then crossed to MMTVNeu transgenic mice, a fifth cross to FVB, to yield control and experimental animals. Mice were fed a conventional diet ad libitum and were maintained at 21 to $22^{\circ} \mathrm{C}$ with a 12-hour light and 12-hour dark cycle.

Animal protocols were approved by the Animal Care and Use Committee of Baylor College of Medicine, and were conducted in accordance with the provisions of the Guide for the Care and Use of Laboratory Animals and the Animal Welfare Act.

Mice were monitored weekly by palpation for tumor induction. Individual tumor size and location was recorded. Twenty p190B+/+/MMTV-Neu littermates and 15 p190B+/-/MMTVNeu littermates were analyzed, and Kaplan-Meier analysis was performed to determine tumor-free survival. Log-rank test analysis was performed to determine whether differences between the two groups were statistically significant.

\section{Tissue preparation}

Heterozygous females (p190B+/- FVB5:C57BI/6) were mated with heterozygous males (MMTV-Neu FVB), with detection of tumors taking place in the F1 generation. Mice at tumor burden $\left(1.0 \mathrm{~mm}^{3}\right)$ were given an intraperitoneal injection of bromodeoxyuridine $(100 \mathrm{mg} / \mathrm{kg})$. Two hours later, the tumors, mammary glands, and lungs were dissected and fixed for 2 hours in 4\% paraformaldehyde. Tumor and lung specimens were dehydrated and embedded in paraffin. Gland specimens were defatted with acetone and stained with Neutral Red overnight, followed by clearing with xylene [20]. Whole-mount pictures were taken using a stereomicroscope (Leica, 
Bannockburn, IL, USA; Model M165 C). After imaging, wholemounted mammary glands were paraffin embedded. Fivemicrometer serial sections were cut into the frontal plane for subsequent histological and immunostaining analysis.

\section{Preparation of tumor lysates and western blotting}

Protein lysates were prepared as previously described [9]. Protein concentrations in the tissue extracts, $\mathrm{p} 190 \mathrm{~B}^{+/+} \mathrm{Neu}(\mathrm{n}$ $=5)$ or $\mathrm{p} 190 \mathrm{~B}^{+/-\mathrm{Neu}}(\mathrm{n}=5)$, were determined using the BCA Protein Quantitation Assay (Pierce, Rockford, IL, USA). Extracts were pooled ( $20 \mu \mathrm{g}$ of each), electrophoresed on $6 \%$ or $12 \%$ SDS-PAGE gels, and transferred to polyvinylidene fluoride (PVDF) membrane (Millipore, Bedford, MA, USA). Membranes were blocked in 5\% milk/Tris-buffered saline Tween20 followed by incubation with p190B 1:1,000 antibody (BD Bioscience, San Jose, CA, USA), ErbB2/Neu 1:1,000 (Neomarkers, Fremont, CA, USA), ErbB3 1:1,000 (Santa Cruz, Santa Cruz, CA, USA), ERK 1:1,000 (Cell Signaling, Danvers, MA, USA), phospho-p21-activated kinase (PAK) 1/2 1:1,000 (Cell Signaling), and $\beta$-actin 1:5,000 (Sigma, St Louis MO, USA) in 5\% milk/Tris-buffered saline Tween-20. Other western blot assays were completed and developed as previously described [9].

\section{Immunohistochemistry and quantification of staining}

Immunohistochemistry was carried out as previously reported [9] using cleaved caspase 3 (9961; Cell Signaling), 1:200 in $5 \%$ BSA, 0.5\% blocking buffer; biotin-conjugated bromodeoxyuridine (550803; BD Pharmingen), 1:10 in 5\% BSA, 0.5\% blocking buffer; and TBS-1 (NeoMarkers), 1:75 in MOM block (Vector Laboratories, Burlingame, CA, USA). Immunofluorescence for von Willebrand factor (VWF) was performed according to the manufacturer's protocol using anti-VWF (DAKO, Glostrup Denmark), 1:200 in 5\% BSA, 0.5\% blocking buffer.

Two independent observers blinded to the experimental groups assessed the peritumoral vascular density by counting the number of vessels within three $100 \times$ fields from each sample. Quantification of TBS-1 staining was performed by counting the number of TBS-1-positive cells in five fields from one gland from each of five mice per genotype.

RNA extraction and quantitative real-time PCR RNA was isolated from briefly digested (collagenase $2 \mathrm{mg} / \mathrm{ml}$ for 1 hour) mammary glands to remove adipose tissue of

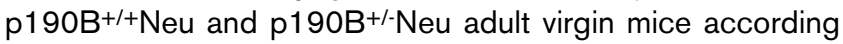
to the manufacturer's protocol (SABiosciences, Frederick, MD, USA). Briefly, Trizol (Invitrogen, Carlsbad, CA, USA) solution was used to according to the manufacturer's protocol followed by an additional purification step using the RNA easy kit (Qiagen, Germantown, MD, USA). The expression of mRNAs involved in the angiogenesis pathway was determined using real-time PCR and a SuperArray Profiler PCR array (SABiosciences, Frederick, MD, USA).

For quantification, cDNA was synthesized using $1 \mu \mathrm{g}$ RNA in a reverse transcription reaction (SABiosciences). cDNA was amplified using the SYBR Green qPCR Master Mix (SABiosciences) with a real-time PCR Applied Biosystems 7500 thermocycler (Applied Biosystems Inc, Foster City, CA, USA). Samples were run in triplicate.

Manufacturer-supplied primer pairs were used to measure mRNAs expressed in the angiogenesis pathway (PAMM-024; SABiosciences) as described in the manufacturer's protocol. The mRNA expression levels were normalized to the expression level of $4 / 5$ housekeeping genes included in the array, excluding $\beta$-actin. The positive threshold was determined based on negative controls as described in the manufacturer's protocol. The calculations for determining relative gene expression were made using the cycle threshold method and web-based PCR array data analysis (SABiosciences).

\section{GTPase activity assays}

Activities of Rho family GTPases were measured using luminescent (RhoA and Rac1) or colorimetric (Cdc42) G-LISA assays (Cytoskeleton, Denver, CO, USA) according to the manufacturer's instructions. Frozen tumors were pulverized using a mortar and pestle, and were homogenized in lysis buffer containing protease inhibitors (Cytoskeleton) using a needle and syringe. The lysates were centrifuged for 2 minutes at $4^{\circ} \mathrm{C}$ and $14,000 \times g$, and the clarified lysates were aliquoted, snap-frozen in liquid nitrogen, and stored at $-80^{\circ} \mathrm{C}$.

The protein concentration was determined using the protein assay reagent provided. Immediately before the assays, the lysates were thawed and the protein concentrations were equalized $(0.75 \mathrm{mg} / \mathrm{ml}$ for RhoA and Rac1, $2.0 \mathrm{mg} / \mathrm{ml}$ for Cdc42) using lysis buffer. For the luminescent assays, SuperSignal West Dura chemiluminescent substrate (Thermo Scientific, Rockford, IL, USA) was substituted for the kit substrate, and a Kodak Gel Logic 1500 digital imaging system and Kodak 1D software (Carestream Health, New Haven, CT, USA) were used to determine the luminescent intensity. For the colorimetric assay, a SpectraMax Plus 384 spectrophotometer and SoftMax Pro software (Molecular Devices, Sunnyvale, CA, USA) were used to determine the absorbance. Means were compared using a $t$ test in GraphPad Prism software (GraphPad Software, Inc., La Jolla, CA, USA).

\section{Mammary tumor tissue transplantation}

Three week-old SCID/Beige mice (Charles River, Germantown, MD, USA) were employed as hosts for transplantation. Both of the Number 4 inguinal mammary glands were cleared of endogenous epithelium. A single tumor piece $\left(1 \mathrm{~mm}^{3}\right.$ in size) was implanted into an incision made in the cleared fat pad of the recipient gland. Mice were monitored weekly by pal- 
pation for tumor induction. The individual tumor size was recorded. Mice were euthanized when tumors reached 10 $\mathrm{mm}^{3}$ in size and were analyzed as described above. For these studies, fragments from five tumors per genotype were each transplanted into 40 recipients. A Fisher's exact test was used to evaluate whether there was a statistically significant difference in transplantation take rates between the two groups.

For reciprocal transplants, 5-week old p190B+/+Neu mice and

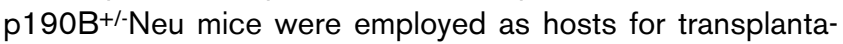
tions. Both of the Number 4 glands were cleared of endogenous epithelium. A single tumor piece $\left(1 \mathrm{~mm}^{3}\right.$ in size) was implanted into an incision made in the cleared fat pads of the recipient gland. Mice were monitored weekly by palpation for tumor induction, and the individual tumor size was recorded. Mice were euthanized when the total tumor burden reached $15 \mathrm{~mm}^{3}$ in size and were analyzed as described above. For these studies, fragments from three tumors per genotype were transplanted into both Number 4 cleared glands of three recipient mice.

\section{Results \\ Haploinsufficiency for p190B inhibits MMTV-Neu tumorigenesis}

To examine whether $\mathrm{p} 190 \mathrm{~B}$ haploinsufficiency affects mammary tumorigenesis, we first backcrossed p190B heterozygous mice $(\mathrm{C} 57 \mathrm{Bl} / 6)$ into the FVB background to circumvent effects of strain-specific modifiers present in the C57BI/6 background that have been reported to delay mammary tumorigenesis [21]. We previously reported that $\mathrm{p} 190 \mathrm{~B}$ heterozygosity resulted in a transient delay in mammary gland development between 4 and 6 weeks of age, but that subsequent mammary gland development and function was unaffected [8]. P190B heterozygous mice (C57BI/6/FVB5) were then crossed with MMTV-Neu transgenic mice [18] to ascertain whether haploinsufficiency of p190B would have any effect on tumor initiation, progression and/or metastasis. The virgin offspring from these crosses were monitored by weekly palpation beginning at 3 months of age and were followed for 18 months.

Strikingly, p190B ${ }^{+/-N e u}$ mice developed tumors with a markedly increased latency compared with the MMTV-Neu mice (74 weeks vs. 35 weeks, $P<0.0001$; Figure 1a), In addition, p190B haploinsufficiency significantly reduced tumor penetrance, in that $53 \%(8 / 15)$ of the heterozygous mice had palpable tumors as compared with $100 \%(20 / 20)$ of the MMTVNeu wild-type mice $(P<0.001)$. Immunoblotting using tumor extracts confirmed that the levels of $\mathrm{p} 190 \mathrm{~B}$ were lower in the p190B ${ }^{+/-N e u}$ tumors, as expected (Figure 1b). Haploinsufficiency for $\mathrm{p} 190 \mathrm{~B}$ also decreased tumor multiplicity (Figure 1c). To determine whether ErbB2 signaling was changed in the p190B ${ }^{+/-N e u}$ tumors, we examined the levels of ErbB2/ $\mathrm{Neu}$ and ErbB3; these levels were similar between the



 histology and growth, but differential signaling}

Histological examination showed that, similar to wild-type MMTV-Neu mice, p190B+/-Neu mice formed adenocarcinomas with grossly similar histopathologies. There appeared to be, however, fewer large blood vessels in the p190B heterozygous tumors as compared with the wild-type tumors (Figure 2a). The $\mathrm{p} 190 \mathrm{~B}^{+/+} \mathrm{Neu}$ and $\mathrm{p} 190 \mathrm{~B}^{+/-N e u}$ tumors had similar growth patterns after initial palpation (Figure 2b). Examination of bromodeoxyuridine incorporation and cleaved caspase 3 as markers of S-phase and apoptosis, respectively, revealed no

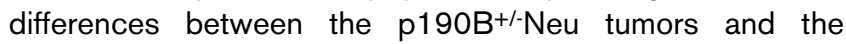
p190B ${ }^{+/+N e u}$ tumors (data not shown).

Western blot analysis of downstream effectors of Rho, how-


ferent Rho signaling patterns as compared with the $\mathrm{p}^{190 \mathrm{~B}^{+/-}}$

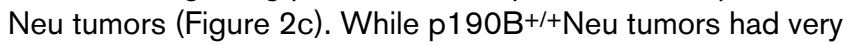
little tumor-to-tumor variation, the $\mathrm{p} 190 \mathrm{~B}^{+/-N} \mathrm{Neu}$ tumors exhibited variable expression of several of the downstream signaling molecules. One notable exception was that a consistent decrease in phosphorylated ROK was detected in the p190B ${ }^{+/-N e u}$ tumors. Some of the heterozygous tumors also exhibited a decrease in total ROK. Interestingly, the levels of PAK1/2 were elevated in the p190B $^{+/-N e u}$ tumors. We also examined the levels of active Rac1, RhoA, and Cdc42. Rac1 was significantly decreased in the heterozygous tumors while RhoA and Cdc42 were unaltered (Figure 2d). These data show that heterozygosity for $\mathrm{p} 190 \mathrm{~B}$ does not affect tumor histology or growth, but does affect Rho signaling pathways.

\section{Haploinsufficiency for p190B blocks preneoplastic progression by reducing angiogenesis}

To determine how p190B haploinsufficiency was inhibiting tumor progression we examined using whole-mount analysis the remaining mammary glands from mice that had developed tumors. These glands exhibited many abnormalities, including enlarged ducts, alveolar-like development, and preneoplastic lesions (Figure 3a). The number of preneoplastic lesions in one number 4 inguinal gland from each mouse at the time of sacrifice for tumor burden was quantified. This analysis

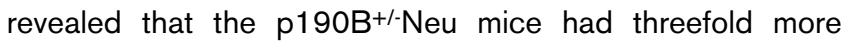

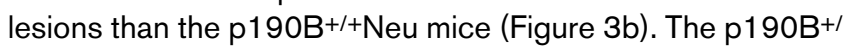
$+\mathrm{Neu}$ lesions were more proliferative than the $\mathrm{p} 190 \mathrm{~B}^{+/-\mathrm{Neu}}$ lesions by bromodeoxyuridine incorporation, but no differences in apoptosis were detected (data not shown).

Angiogenesis is a key step in the progression of preneoplastic lesions ( 1 to $2 \mathrm{~mm}$ in size) to overt tumors [22]. We therefore considered the possibility that a block in the angiogenic switch may be one potential mechanism by which haploinsufficiency for $\mathrm{p} 190 \mathrm{~B}$ resulted in an increased number of preneoplastic lesions, but fewer tumors. To examine the gene expression of potential mediators of the angiogenic switch, mRNA isolated




Figure 1

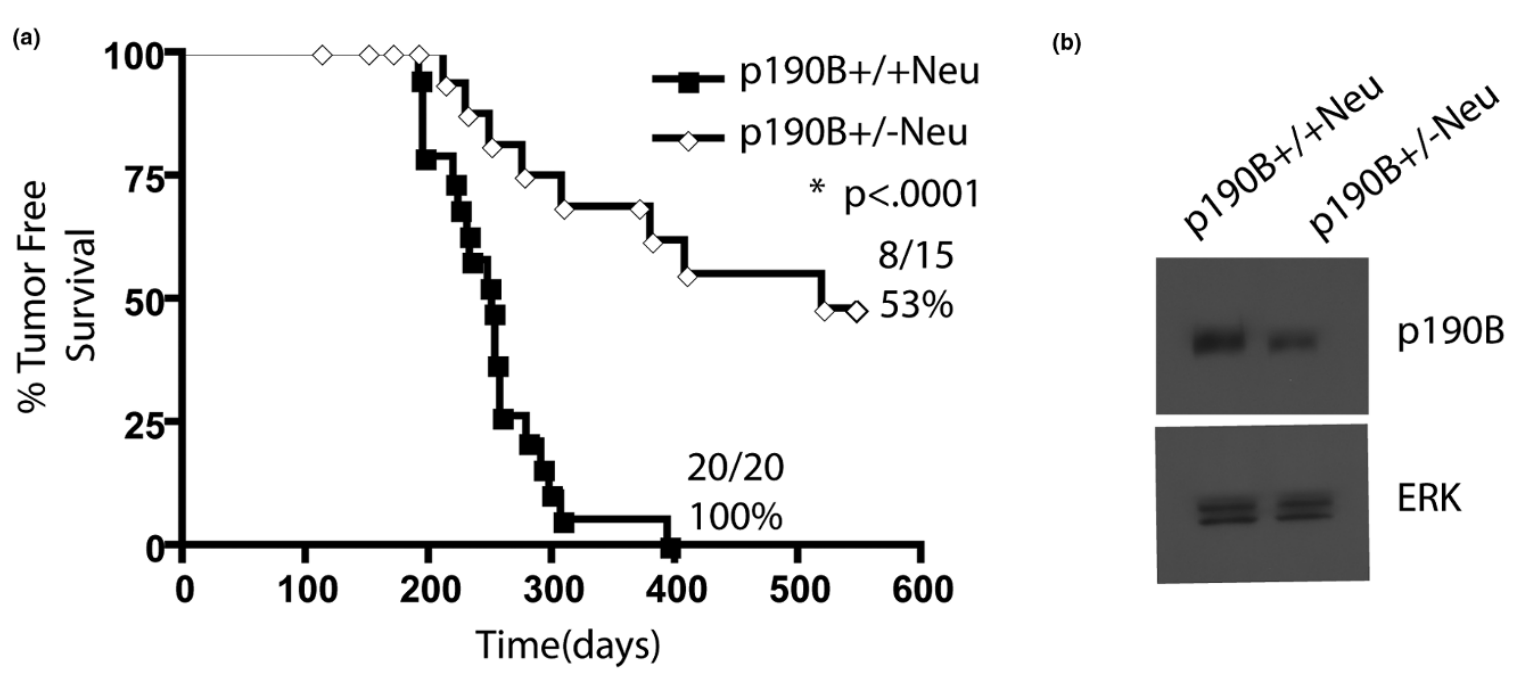

(c)

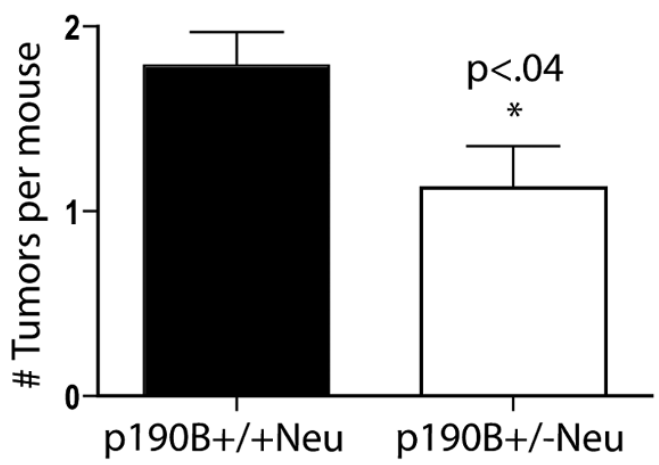

(d)

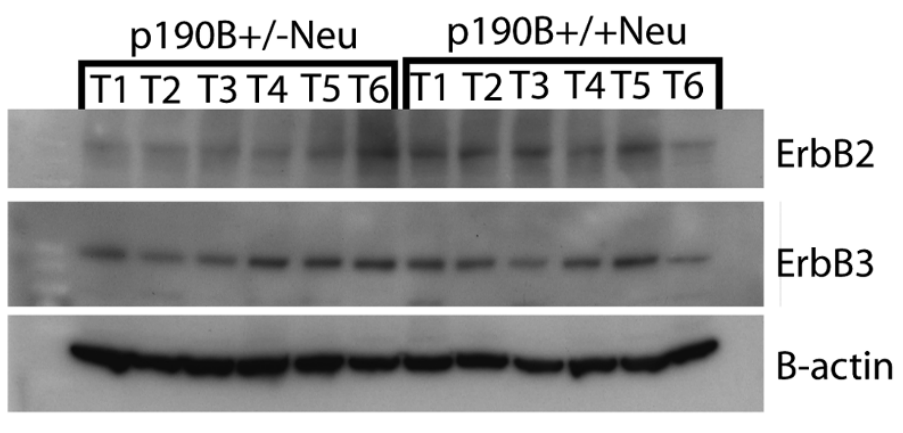

Haploinsufficiency for p190B delays MMTV-Neu tumor onset and causes a decrease in tumor multiplicity. (a) Kaplan-Meier tumor-free survival curve

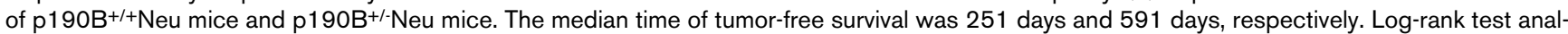
ysis showed a significant difference between the two groups $(P<0.0001)$. (b) Immunoblotting for $\mathrm{p} 190 \mathrm{~B}$ and ERK in pooled tumor tissue from five tumors of indicated genotypes. (c) Tumor multiplicity for $\mathrm{p} 190 \mathrm{~B}^{+/+}$Neu mice and for $\mathrm{p} 190 \mathrm{~B}^{+/-N e u}$ mice $(1.8$ and 1.2 tumors per mouse, respectively). Student's $t$ test showed a significant decrease $(P<0.04)$. (d) Immunoblotting for ErbB2, ErbB3, and $\beta$-actin in six individual tumors from the indicated genotypes. Error bars, standard error of the mean.

Neu virgin mammary glands was analyzed using an angiogenesis Superarray platform. While few significant changes in expression of these genes were detected, we observed a twofold increase in the mRNA expression of TBS-1 (Figure 3c and Additional data file 1), a potent angiogenic inhibitor produced by stromal fibroblasts, endothelial cells and immune cells. Furthermore, quantification of immunostaining to detect TBS-1 protein in mammary gland sections demonstrated that expression of TBS-1 was elevated twofold in the p190-B ${ }^{+/-N e u}$ glands as compared with the wild-type glands (Figure $3 d, e$ ). These data suggest that $\mathrm{p} 190 \mathrm{~B}$ haploinsufficiency delays preneoplastic progression through inhibition of the angiogenic switch.

\section{Loss of p190B results in decreased tumor vasculature and fewer lung metastases}

To determine whether this inhibition of angiogenesis is also



vessels using immunostaining for an endothelial specific marker (VWF) (Figure 4b). This analysis showed a twofold decrease in the number of vessels in the $\mathrm{p}^{190 \mathrm{~B}^{+/-} \mathrm{Neu} \text { tumors }}$


also examined the lungs of $\mathrm{p} 190 \mathrm{~B}^{+/-\mathrm{Neu}}$ and $\mathrm{p} 190 \mathrm{~B}^{+/+} \mathrm{Neu}$ mice with similar tumor burdens to determine whether $\mathrm{p} 190 \mathrm{~B}$ haploinsufficiency affected metastasis. The percentage of mice with metastases was not significantly different between the two genotypes due to the limited number of $\mathrm{p} 190 \mathrm{~B}$ heterozygous mice that exhibited primary tumors. The $\mathrm{p}^{190 \mathrm{~B}^{+/-} \mathrm{Neu}}$


+Neu mice, however, despite the fact that they had similar primary tumor burdens (Figure 4c). These results suggest that haploinsufficiency for $\mathrm{p} 190 \mathrm{~B}$ inhibits tumor angiogenesis and

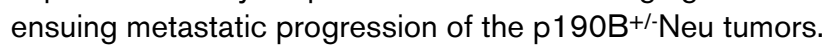


Figure 2

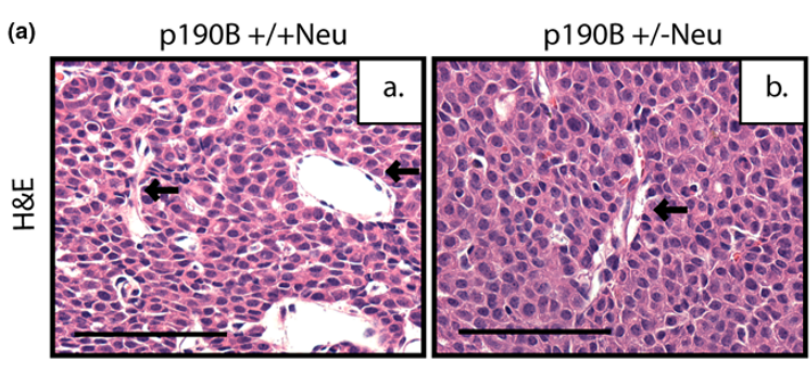

(c)

(b)



p-PAK $1 / 2$

actin





ROK

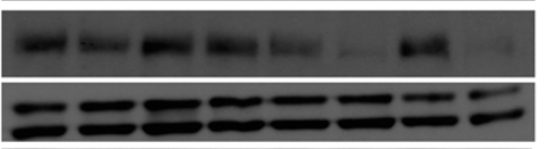

ERK $=2-2=$

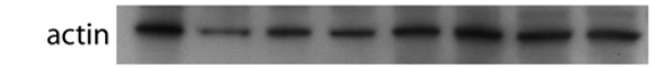

(d)


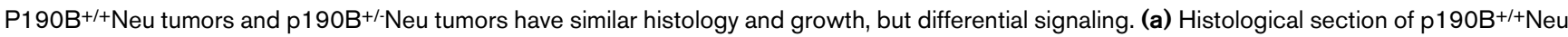
tumors and $\mathrm{p} 190 \mathrm{~B}^{+/-N} \mathrm{Neu}$ tumors stained with hematoxylin and eosin (H\&E). Arrows point to vasculature. Scale bar $=50 \mu \mathrm{m}$. (b) Growth curve of p190B ${ }^{+/+}$Neu tumors and p190B $1 /$-Neu tumors. No statistical difference. (c) Immunoblotting for phosphorylated Rho kinase (pROK), Rho kinase (ROK), phosphorylated p21-activated kinase (p-PAK)1/2, and total ERK and actin as loading controls in four independent tumors from each indicated genotype. Quantification of western blots is graphed. (d) Quantification of active GTPase levels in tumor tissue. Analysis was carried out using Image J software. Error bars, standard error of the mean.

 by wild-type stroma and growth of wild-type tumors is inhibited by heterozygous stroma}

To determine the relative contribution of the epithelium and the stroma to the tumor progression process, tumor pieces from


transplanted into cleared fatpads of SCID/beige mice. Inter-

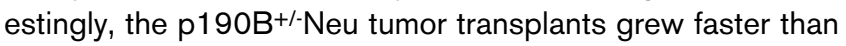

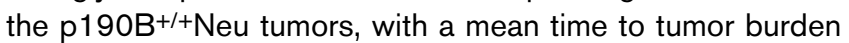
of 89 days compared with 126 days, respectively $(P<$ 0.0001 ; Figure $5 \mathrm{a})$. The transplantation take rates were similar between the two groups (Figure 5b), with no statistical difference determined by the Fisher exact test.

We next analyzed the transplanted tumors to determine whether the vascular defect seen in the original tumors persisted in the transplants. For this analysis, the tumors were stained with VWF and the vessels were counted. Interestingly, no significant differences in the number of large and small vessels were observed in both the $\mathrm{p} 190 \mathrm{~B}^{+/+} \mathrm{Neu}$ and $\mathrm{p} 190 \mathrm{~B}^{+/-}$ Neu tumor transplants (Figure $5 c, d$ ). These data indicate that the effects of p190B haploinsufficiency on MMTV-Neu tumor 


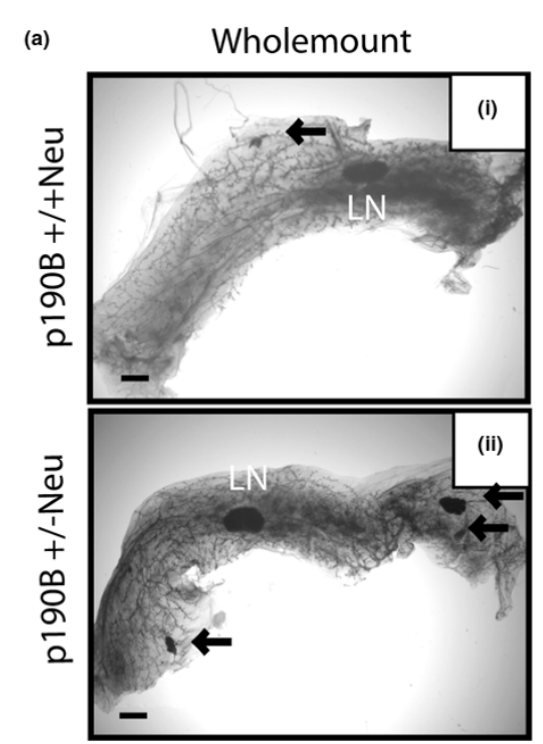

(d)

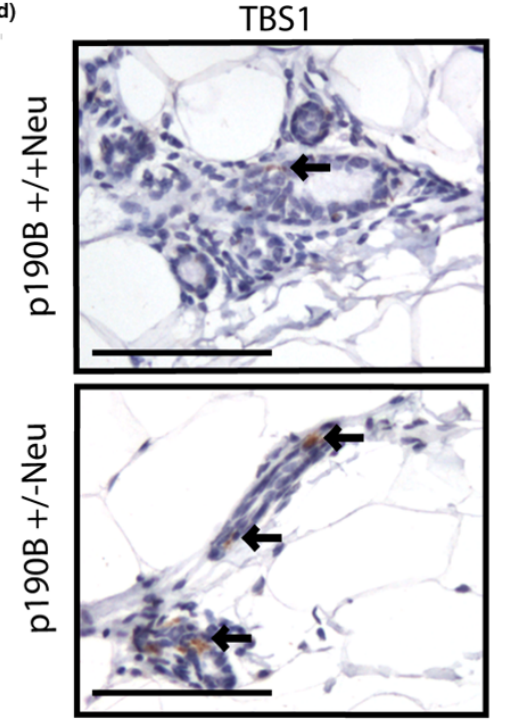

(b)

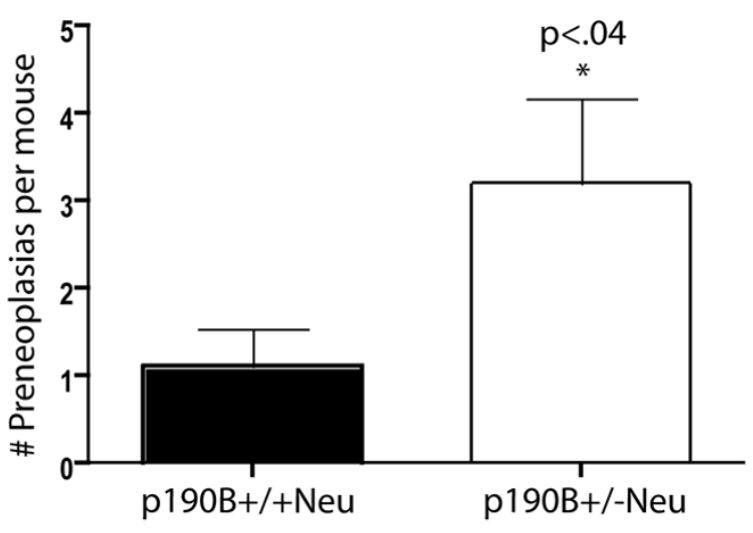

(c)

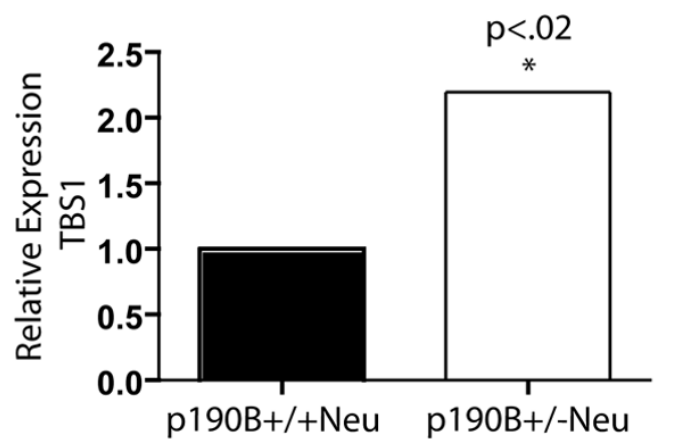

(e)



Haploinsufficiency for $\mathrm{p} 190 \mathrm{~B}$ blocks preneoplastic progression by reducing angiogenesis. (a) Mammary tissues from tumor burden mice with the indicated genotypes were examined by whole-mount analyses. Arrows, preneoplasias detected. LN, lymph node. Scale bar $=500 \mu \mathrm{m}$. (b) Number of preneoplastic lesions in one Number 4 inguinal gland per tumor-burden mouse was quantified. Student's $t$ test showed significant difference between groups $(P<0.04)$. (c) Gene expression qRT-PCR data from Superarray angiogenesis array. Array analysis revealed a twofold increase in thrombospondin-1 (TBS-1) in the p190B +/-Neu mammary glands of adult mice 8 to 12 weeks of age. (d) Immunohistochemistry to detect TBS-1

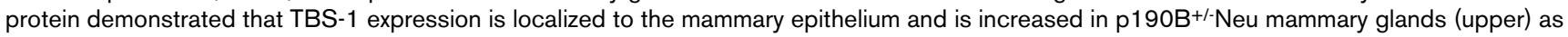
compared with wild-type glands (lower). Images shown are at 400x magnification. (e) Quantification of TBS-1 immunostaining showed that TBS-1 protein is increased fourfold in $\mathrm{p} 190 \mathrm{~B}^{+/-}$Neu mammary glands. Error bars, standard error of the mean.

angiogenesis are due to defects in the vasculature and/or stroma.

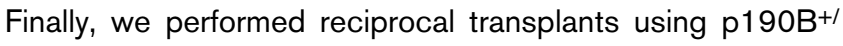
+Neu and p190B+/-Neu 5-week-old mice as the host to examine the contribution of the heterozygous stroma. The $190 \mathrm{~B}^{+1}$ -Neu tumor pieces all grew to form tumors in the $\mathrm{p}_{190 \mathrm{~B}^{+/+} \mathrm{Neu}}$ stroma $(n=6)$, while none of the $\mathrm{p}_{190 \mathrm{~B}^{+/+}+\mathrm{Neu} \text { tumor pieces }}$



stroma $(n=6)$. These data further support p190B haploinsufficiency in the vasculature and/or stroma as being responsible for the tumor growth potential.

\section{Discussion}

In the present article we show that p190B RhoGAP, a gene that is essential for mammary gland development, plays a critical role in MMTV-Neu mammary tumor progression. Haploinsufficiency for $\mathrm{p} 190 \mathrm{~B}$ increases tumor-free survival, reduces 
Figure 4

(a)

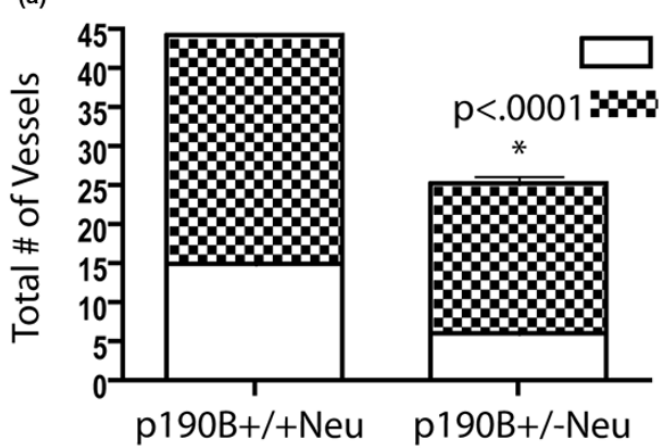

(c)

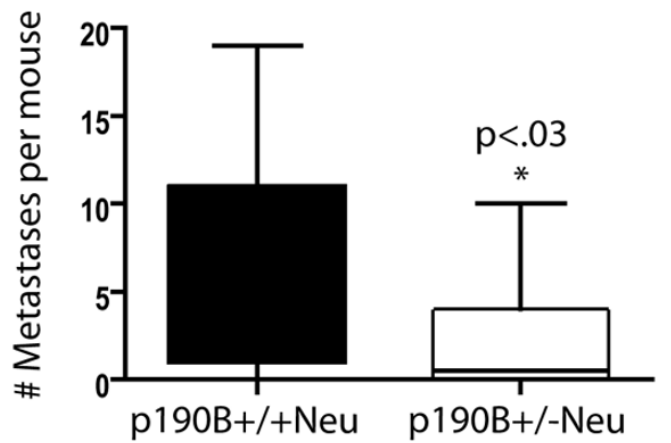

(b)



Loss of $\mathrm{p} 190 \mathrm{~B}$ results in decreased tumor vasculature and fewer lung metastases. (a) Number of vessels per $100 \times$ section of tumor stained with von Willebrand factor (VWF) was quantified. Student's $t$ test shows a significant difference between groups $(P<0.0001)$. (b) Representative pictures of VWF immunofluorescence (red) and dapi (blue) in the two different genotypes as indicated. Arrows mark blood vessels. Scale bar $=100$ $\mu \mathrm{m}$. (c) Number of metastases per mouse at tumor burden, as determined by counting metastatic nodules in serial sections of the entire lung. Stu-

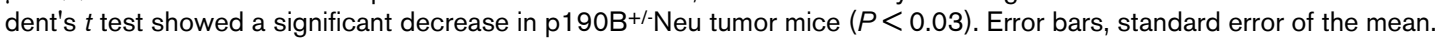

tumor penetrance, and decreases tumor multiplicity. The level of Neu transgene expression is unaltered in the $\mathrm{p}^{190 \mathrm{~B}^{+/-} \mathrm{Neu}}$ normal mammary tissue and tumors, and thus downregulation of transgene expression is not responsible for the inhibition of tumor progression. ErbB3 expression was also similar between the two groups, suggesting that p190B deficiency does not inhibit Neu-induced tumorigenesis by altering the epidermal growth factor receptor signaling axis that promotes tumor formation in this model [23]. Interestingly, p190B heterozygosity does not inhibit the growth rate of established tumors. Furthermore, we found in a few of the $\mathrm{p}^{190 \mathrm{~B}^{+/-} \mathrm{Neu}}$ tumors that $\mathrm{p} 190 \mathrm{~B}$ expression levels were comparable with


shown).

It has been reported that GAPs and related $\mathrm{G}$ proteins are upregulated in MMTV-Neu tumors [19], and our results confirm that this signaling network plays an important role in MMTV-Neu tumor progression. Based on previous studies using p190B-deficient mouse embryonic fibroblasts [12], we anticipated that reduced $\mathrm{p} 190 \mathrm{~B}$ expression in vivo would increase Rho GTPase activities as well as signaling through the downstream effectors ROK and PAK. GTPase assays to examine the levels of active RhoA, Rac1, and Cdc42, however, demonstrated that only Rac1 levels were altered in the heterozygous tumors, and they were in fact decreased. In addition, ROK activity - and in some cases ROK expression levels were reduced in the p190B-deficient tumors, whereas PAK1/ 2 activity levels were increased. These data indicate that $\mathrm{p} 190 \mathrm{~B}$ is required for proper regulation of Rho/ROK signaling during Neu mammary tumor progression.

Rac1 and PAK activities have been shown to play important roles in mammary tumorigenesis $[24,25]$, and thus PAK1/2 activities may be elevated in the p190B-deficient mice to compensate for the decrease in Rac1 activity. It is interesting, and perhaps not surprising, that $\mathrm{p} 190 \mathrm{~B}$ deficiency does not result in persistent upregulation of Rho GTPase activity in the tumors. Rho GTPases play critical roles in essential cellular processes such as cell cycle progression, mitosis, and cell survival, and GTPase activity is tightly controlled during these processes [26]. Downstream effectors such as PAK and ROK are also critical for these processes $[27,28]$. A loss of p190B resulting in persistently elevated activity of several Rho 
(a)

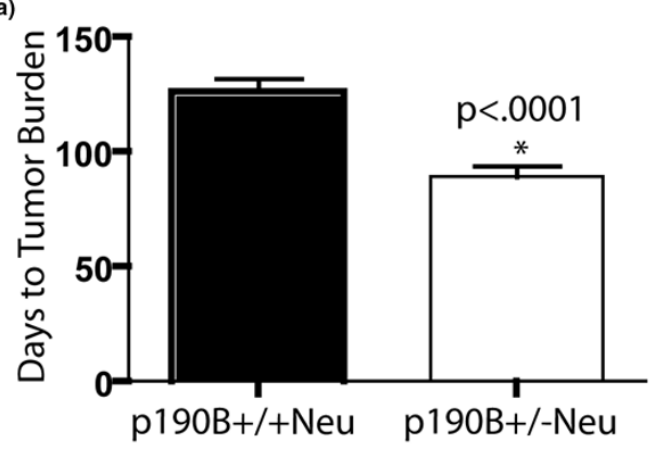

(b)

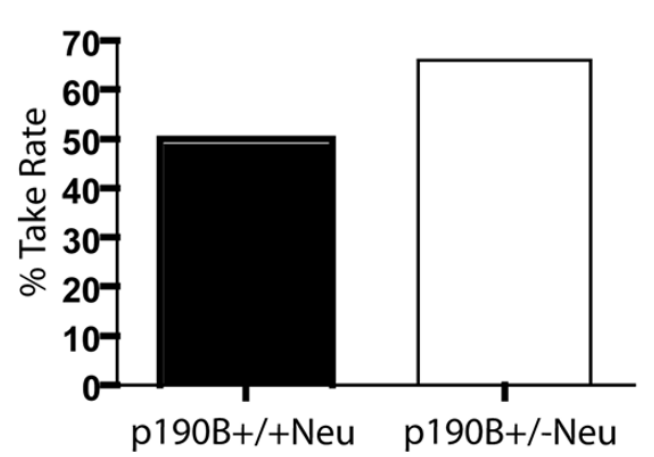

(d)

(d) VWF (c)

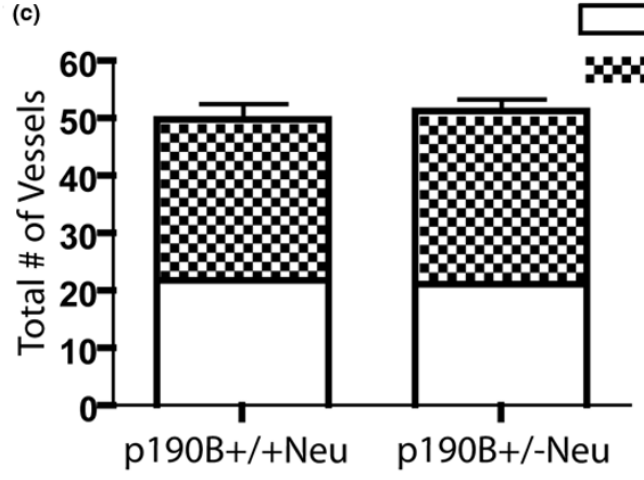

(e)

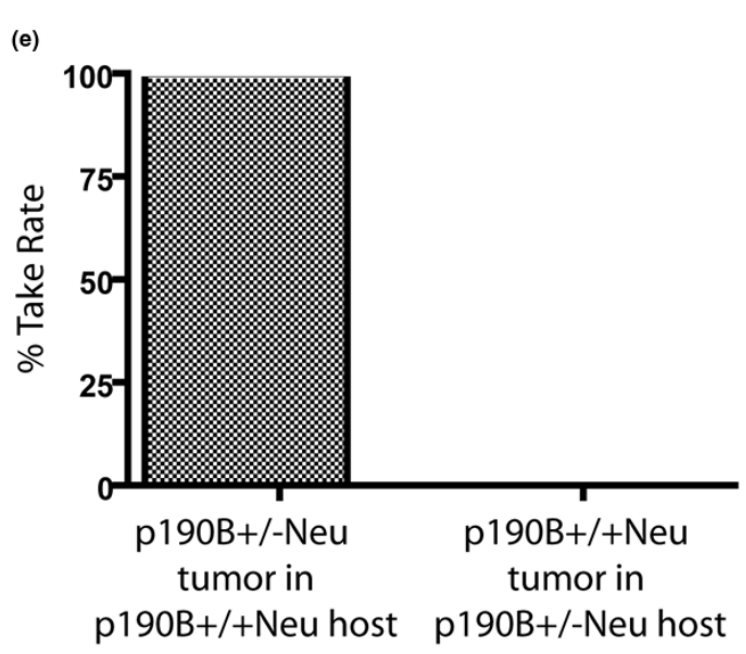

Large Vessels $\omega$ Small Vessels




ignated genotype tumor piece into the cleared fatpad of a SCID/Beige mouse. Decreased time to tumor burden was statistically significant in

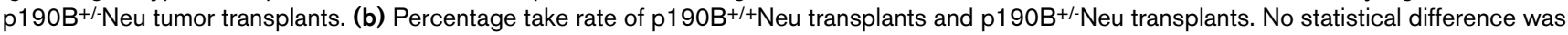
seen between genotypes. (c) Number of vessels per 100x section of tumor stained with von Willebrand factor (VWF) was quantified. No statistical difference between genotypes was detected. (d) Representative pictures of VWF immunofluorescence (red) and dapi (blue) in the two different genotypes as indicated. Arrows mark blood vessels. Scale bar $=100 \mu \mathrm{m}$. (e) Percentage take rate of $\mathrm{p} 190 \mathrm{~B}^{+/-N e u}$ tumors into a p190B ${ }^{+/+N e u}$ host and of $\mathrm{p} 190 \mathrm{~B}^{+/+} \mathrm{Neu}$ tumors into a $190 \mathrm{~B}^{+/-} \mathrm{Neu}$ host. The $\mathrm{p} 190 \mathrm{~B}^{+/-N} \mathrm{Neu}$ tumors had a $100 \%$ take rate, while the $\mathrm{p} 190 \mathrm{~B}^{+/+} \mathrm{Neu}$ tumors had a $0 \%$ take rate. Statistically significant difference $P<0.0001$. Error bars, standard error of the mean.

GTPases is therefore likely to have a negative impact on cell survival or proliferation. Other GAPs or guanine nucleotide dissociation inhibitors may be upregulated or the guanine nucleotide exchange factor activity may be downregulated to compensate for the loss of p190B. In some tumors, we noted that $\mathrm{p} 190 \mathrm{~B}$ levels were similar to levels in wild-type tumors, suggesting compensatory mechanisms were functioning to restore $\mathrm{p} 190 \mathrm{~B}$ levels. 
Taken together, these results demonstrate that p190B deficiency results in unexpected effects on the Rho signaling axis and reveal an important role for $\mathrm{p} 190 \mathrm{~B}$ in regulating these signaling pathways during mammary tumor progression. In addition, these results highlight the importance of investigating the role of these signaling pathways in tumorigenesis in the context of the complex in vivo environment.

Examination of the mammary glands from the $\mathrm{p}^{190 \mathrm{~B}^{+/-} \mathrm{Neu}}$ tumor-burden mice indicated that there were a higher number of preneoplastic lesions. This increase probably resulted from a lack of progression, since averaging the number of preneoplastic lesions and the number of tumors showed no statistical difference in the total number of initiated lesions between the

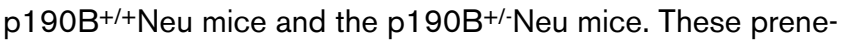
oplastic lesions were characteristic of the avascular phase of the angiogenic switch, as they were approximately 1 to $2 \mathrm{~mm}$ in diameter $[22,29]$. Interestingly, several $\mathrm{p} 190 \mathrm{~B}^{+/-\mathrm{Neu}}$ mice did not develop tumors by 18 months of age and, when their glands were examined for preneoplastic lesions, no lesions were detected (7/7 mice) (data not shown). These data suggest that $\mathrm{p} 190 \mathrm{~B}$ is important for the progression of preneoplastic lesions, but that it may also play a role in tumor initiation. Further studies in which normal mammary tissue pieces from

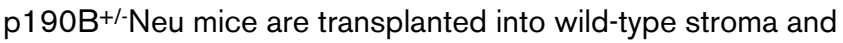
are allowed to proceed through the stochastic process of tumor formation will be required to examine preneoplastic lesions in more detail.

The possibility that $\mathrm{p} 190 \mathrm{~B}$ was important for the progression of avascular preneoplastic lesions to vascularized lesions prompted us to examine angiogenesis in the $\mathrm{p}^{190 \mathrm{~B}^{+/-} \mathrm{Neu}}$ mice. To characterize the potential for a vascular defect in these mice we utilized the Superarray qRT-PCR angiogenesis platform, which allowed us to examine the expression of 87 angiogenesis genes, both positive and negative regulators, including vascular endothelial growth factor, matrix metalloproteinase-2 and matrix metalloproteinase-9, hypoxia inducible factor 1 alpha, fibroblast growth factor genes, TBS-1, and transforming growth factor beta genes. Only TBS-1 was significantly increased within the adult virgin mammary glands of


for TBS-1 confirmed this increase.

Previous studies have demonstrated that TBS- 1 is a component of the extracellular matrix that functions as a negative regulator of tumor vasculature, and TBS-1 expression dramatically effects mammary tumorigenesis. For example, overexpression of TBS-1 inhibited angiogenesis in transgenic mice overexpressing activated $\mathrm{Neu}$, resulting in delayed tumor onset and reduced tumor penetrance [30]. Conversely, TBS1 deficiency in the transgenic mice overexpressing activated $\mathrm{Neu}$ increased angiogenesis, enhanced growth, and reduced tumor latency [30].
TBS-1 has previously been linked to GTPase signaling. Ras/ Rho signaling has been shown to repress TBS-1 through effects on phosphatidyl inositol-3 kinase [31], and Ras/Rhomediated suppression of TBS-1 has been suggested as necessary to promote angiogenesis [32]. Disruption of Rho/ROK signaling may therefore account for the elevated TBS-1

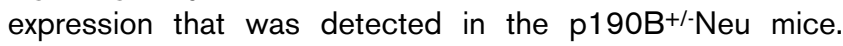
Increased expression of this potent angiogenic inhibitor may be responsible, in part, for the dramatic inhibition of tumorigenesis that occurred in the $\mathrm{p}^{190 \mathrm{~B}^{+/-} \mathrm{Neu} \text { mice. }}$

Consistent with the increased TBS-1 expression in $\mathrm{p}^{190 \mathrm{~B}^{+/-}}$ Neu mammary glands, p190B haploinsufficiency also resulted in decreased angiogenesis in the tumors. This persistent vascular defect may be responsible for the decreased number of metastatic nodules detected in the tumor-bearing $\mathrm{p} 190 \mathrm{~B}$ heterozygous mice. Interestingly, an increased vascular density has been correlated with poor breast cancer prognosis, and TBS-1 expression was inversely correlated with malignant progression of mammary and lung carcinomas as well as melanoma [33,34].

Our transplantation experiments showed that the reduction in angiogenesis was probably due to defects in the stroma or vasculature because transplantation of $\mathrm{p}^{190 \mathrm{~B}^{+/-} \mathrm{Neu}}$ tumor pieces into wild-type stroma restored angiogenesis to the levels detected in wild-type tumors. Conversely, wild-type tumors pieces failed to grow in the p190B-deficient stroma, suggesting that loss of $\mathrm{p} 190 \mathrm{~B}$. These data are consistent with recent studies in which siRNA knockdown of p190B inhibited capillary tube formation in Matrigel by human umbilical vein endothelial cells [35]. Interestingly, these transplantation experiments revealed a decreased time to tumor burden in the


+ Neu tumors. The mechanism responsible for this difference remains to be elucidated. One potential explanation, however, may be that the cells which initiated the $\mathrm{p} 190 \mathrm{~B}^{+/-N e u}$ tumors had already acquired significant genetic changes in order to develop the initial tumor in the absence of one allele of $\mathrm{p} 190 \mathrm{~B}$, which in the presence of wild-type stroma impart a growth advantage. For example, PAK1/2 activity is elevated in the heterozygous tumors, and PAK1 hyperactivation promotes mammary tumor formation in part by activating p38MAPK signaling and proliferation [25]. Elevated PAK activity may therefore allow the tumor cells to respond more robustly to proliferative cues present in the wild-type stroma. Further studies, including microarray analysis of the original and transplanted tumors, as well as transplantation experiments in which $\mathrm{p}^{190 \mathrm{~B}^{+/-} \mathrm{Neu}}$ mammary epithelium is introduced into wild-type stroma and is allowed to go through the stochastic process of tumor formation, will be required to fully understand the contribution of p190B in the epithelium to tumor initiation, growth, and progression. 


\section{Conclusions}

Elevated expression of Rho GTPases has been detected in many types of cancer including breast cancer [36]. RhoA, RhoB, Rac1/Rac1b, and Cdc42 are overexpressed in breast tumors, with RhoA expression associated with advanced stages of the disease [6,37]. RhoC overexpression has been associated with inflammatory breast cancer and may be involved in tumor angiogenesis [5,7]. Our studies demonstrate that $\mathrm{p} 190 \mathrm{~B}$, an inhibitor of the Rho GTPases, is required for Rho/ROK signaling during mammary tumorigenesis and point to a critical role for $\mathrm{p} 190 \mathrm{~B}$ in the vasculature during tumor progression. These studies highlight the importance of investigating the effects of altered Rho signaling in the context of the in vivo environment in order to elucidate the role of this signaling network in breast cancer. The functional role of Rho GTPases in the different subtypes of human breast cancer remains understudied, but these studies using mouse models suggest that this pathway may play an important role in tumor progression.

\section{Competing interests}

The authors declare that they have no competing interests.

\section{Authors' contributions}

$\mathrm{BMH}-\mathrm{S}$ performed the majority of the experiments described in the paper, drafted the manuscript, and contributed to the intellectual development of the study. TV-G performed transplantation experiments, co-drafted the manuscript, and contributed to the study design, data analysis and interpretation. PRM quantified and interpreted the GTPase assays. VJ quantified and interpreted metastasis data. MPH assisted with transplantations, performed VWF and TBS-1 immunostaining, and quantified and interpreted these data. SGH participated in the design of the study and performed statistical analysis. JS provided p190B knockout mice and participated in the design and interpretation of the study. JMR directed the design and implementation of the study, interpreted data, and co-drafted the manuscript. All authors read and approved the final manuscript.

\section{Additional files}

The following Additional files are available online:

\section{Additional file 1}

A Word file containing a table showing the fold changes and $P$ values for the angiogenesis superarray comparing p190B ${ }^{+/-N e u ~ m a m m a r y ~ g l a n d s ~ w i t h ~} \mathrm{p}^{190 \mathrm{~B}^{+/+} \mathrm{Neu}}$ mammary glands.

See http://www.biomedcentral.com/content/

supplementary/bcr2352-S1.doc

\section{Acknowledgements}

These studies were supported through grant CA030195-22. TV-G is funded by K99CA127361. BMH-S was funded by DOD predoctoral fellowship W81XWH-06-1-0704 and is currently a NCl Cancer Prevention Fellow. The authors thank Shirley Small for her help with animal husbandry and colony management, and Maria Gonzalez-Rimbau for technical support.

\section{References}

1. Olofsson B: Rho guanine dissociation inhibitors: pivotal molecules in cellular signalling. Cell Signal 1999, 11:545-554.

2. Bernards A: GAPs galore! A survey of putative Ras superfamily GTPase activating proteins in man and Drosophila. Biochim Biophys Acta 2003, 1603:47-82.

3. Schmidt A, Hall A: Guanine nucleotide exchange factors for Rho GTPases: turning on the switch. Genes Dev 2002, 16:1587-1609.

4. Rihet S, Vielh P, Camonis J, Goud B, Chevillard S, de Gunzburg J: Mutation status of genes encoding RhoA, Rac1, and Cdc42 GTPases in a panel of invasive human colorectal and breast tumors. J Cancer Res Clin Oncol 2001, 127:733-738.

5. van Golen KL, Wu ZF, Qiao XT, Bao L, Merajver SD: RhoC GTPase overexpression modulates induction of angiogenic factors in breast cells. Neoplasia 2000, 2:418-425.

6. Fritz G, Just I, Kaina B: Rho GTPases are over-expressed in human tumors. Int J Cancer 1999, 81:682-687.

7. van Golen KL, Davies S, Wu ZF, Wang Y, Bucana CD, Root H, Chandrasekharappa S, Strawderman M, Ethier SP, Merajver SD: A novel putative low-affinity insulin-like growth factor-binding protein, LIBC (lost in inflammatory breast cancer), and RhoC GTPase correlate with the inflammatory breast cancer phenotype. Clin Cancer Res 1999, 5:2511-2519.

8. Chakravarty G, Hadsell D, Buitrago W, Settleman J, Rosen JM: p190-B RhoGAP regulates mammary ductal morphogenesis. Mol Endocrinol 2003, 17:1054-1065.

9. Vargo-Gogola T, Heckman BM, Gunther EJ, Chodosh LA, Rosen JM: P190-B Rho GTPase-activating protein overexpression disrupts ductal morphogenesis and induces hyperplastic lesions in the developing mammary gland. Mol Endocrinol 2006, 20:1391-1405.

10. Burbelo PD, Miyamoto $S$, Utani A, Brill $S$, Yamada KM, Hall $A$, Yamada Y: p190-B, a new member of the Rho GAP family, and Rho are induced to cluster after integrin cross-linking. J Biol Chem 1995, 270:30919-30926.

11. Bustos RI, Forget MA, Settleman JE, Hansen SH: Coordination of Rho and Rac GTPase function via p190B RhoGAP. Curr Biol 2008, 18:1606-1611.

12. Sordella R, Classon M, Hu KQ, Matheson SF, Brouns MR, Fine $B$, Le Z, Takami H, Yamada Y, Settleman J: Modulation of CREB activity by the Rho GTPase regulates cell and organism size during mouse embryonic development. Dev Cell 2002, 2:553-565.

13. Sordella R, Jiang W, Chen GC, Curto M, Settleman J: Modulation of Rho GTPase signaling regulates a switch between adipogenesis and myogenesis. Cell 2003, 113:147-158.

14. Heckman BM, Chakravarty G, Vargo-Gogola T, Gonzales-Rimbau M, Hadsell DL, Lee AV, Settleman J, Rosen JM: Crosstalk between the p190-B RhoGAP and IGF signaling pathways is required for embryonic mammary bud development. Dev Biol 2007, 309:137-149.

15. Chakravarty G, Roy D, Gonzales M, Gay J, Contreras A, Rosen JM: P190-B, a Rho-GTPase-activating protein, is differentially expressed in terminal end buds and breast cancer. Cell Growth Differ 2000, 11:343-354.

16. Slamon DJ, Clark GM, Wong SG, Levin WJ, Ullrich A, McGuire WL: Human breast cancer: correlation of relapse and survival with amplification of the HER-2/neu oncogene. Science 1987 , 235:177-182.

17. Ursini-Siegel J, Schade B, Cardiff RD, Muller WJ: Insights from transgenic mouse models of ERBB2-induced breast cancer. Nat Rev Cancer 2007, 7:389-397.

18. Guy CT, Webster MA, Schaller M, Parsons TJ, Cardiff RD, Muller WJ: Expression of the neu protooncogene in the mammary 
epithelium of transgenic mice induces metastatic disease. Proc Natl Acad Sci USA 1992, 89:10578-10582.

19. Desai KV, Xiao N, Wang W, Gangi L, Greene J, Powell Jl, Dickson R, Furth P, Hunter K, Kucherlapati R, Simon R, Liu ET, Green JE: Initiating oncogenic event determines gene-expression patterns of human breast cancer models. Proc Natl Acad Sci USA 2002, 99:6967-6972.

20. Moraes RC, Zhang X, Harrington N, Fung JY, Wu MF, Hilsenbeck SG, Allred DC, Lewis MT: Constitutive activation of smoothened (SMO) in mammary glands of transgenic mice leads to increased proliferation, altered differentiation and ductal dysplasia. Development 2007, 134:1231-1242.

21. Henry MD, Triplett AA, Oh KB, Smith GH, Wagner KU: Parityinduced mammary epithelial cells facilitate tumorigenesis in MMTV-neu transgenic mice. Oncogene 2004, 23:6980-6985.

22. Naumov GN, Akslen LA, Folkman J: Role of angiogenesis in human tumor dormancy: animal models of the angiogenic switch. Cell Cycle 2006, 5:1779-1787.

23. Siegel PM, Ryan ED, Cardiff RD, Muller WJ: Elevated expression of activated forms of Neu/ErbB-2 and ErbB-3 are involved in the induction of mammary tumors in transgenic mice: implications for human breast cancer. EMBO J 1999, 18:2149-2164.

24. Strumane K, Rygiel T, Valk M van der, Collard JG: Tiam1-deficiency impairs mammary tumor formation in MMTV-c-neu but not in MMTV-c-myc mice. J Cancer Res Clin Oncol 2009, 135:69-80.

25. Wang RA, Zhang $H$, Balasenthil S, Medina D, Kumar R: PAK1 hyperactivation is sufficient for mammary gland tumor formation. Oncogene 2006, 25:2931-2936.

26. Vega FM, Ridley AJ: Rho GTPases in cancer cell biology. FEBS Lett 2008, 582:2093-2101.

27. Molli PR, Li DQ, Murray BW, Rayala SK, Kumar R: PAK signaling in oncogenesis. Oncogene 2009, 28:2545-2555.

28. Narumiya S, Yasuda S: Rho GTPases in animal cell mitosis. Curr Opin Cell Biol 2006, 18:199-205.

29. Bergers G, Benjamin LE: Tumorigenesis and the angiogenic switch. Nat Rev Cancer 2003, 3:401-410.

30. Rodriguez-Manzaneque JC, Lane TF, Ortega MA, Hynes RO, Lawler J, Iruela-Arispe ML: Thrombospondin-1 suppresses spontaneous tumor growth and inhibits activation of matrix metalloproteinase-9 and mobilization of vascular endothelial growth factor. Proc Natl Acad Sci USA 2001, 98:12485-12490.

31. Watnick RS, Cheng YN, Rangarajan A, Ince TA, Weinberg RA: Ras modulates Myc activity to repress thrombospondin-1 expression and increase tumor angiogenesis. Cancer Cell 2003, 3:219-231.

32. Merajver SD, Usmani SZ: Multifaceted role of Rho proteins in angiogenesis. J Mammary Gland Biol Neoplasia 2005, 10:291-298.

33. Weinstat-Saslow DL, Zabrenetzky VS, VanHoutte K, Frazier WA, Roberts DD, Steeg PS: Transfection of thrombospondin 1 complementary DNA into a human breast carcinoma cell line reduces primary tumor growth, metastatic potential, and angiogenesis. Cancer Res 1994, 54:6504-6511.

34. Weidner N: Tumoural vascularity as a prognostic factor in cancer patients: the evidence continues to grow. J Pathol 1998, 184:119-122.

35. Guegan F, Tatin F, Leste-Lasserre T, Drutel G, Genot E, Moreau V: p190B RhoGAP regulates endothelial-cell-associated proteolysis through MT1-MMP and MMP2. J Cell Sci 2008, 121:2054-2061.

36. Tang Y, Olufemi L, Wang MT, Nie D: Role of Rho GTPases in breast cancer. Front Biosci 2008, 13:759-776.

37. Schnelzer A, Prechtel D, Knaus U, Dehne K, Gerhard M, Graeff H, Harbeck N, Schmitt M, Lengyel E: Rac1 in human breast cancer: overexpression, mutation analysis, and characterization of a new isoform, Rac1b. Oncogene 2000, 19:3013-3020. 